\title{
Enniatin Production by Fusarium sambucinum: Primary, Secondary, and Unitary Metabolism
}

\author{
By T. K. AUDHYA AND D. W. RUSSELL \\ Department of Biochemistry, Dalhousie University, Halifax, Nova Scotia, Canada $\mathrm{B}_{3} \mathrm{H}_{4} \mathrm{H}_{7}$
}

(Received 3 September 1974)

\begin{abstract}
SUMMARY
Fusarium sambucinum liquid surface cultures on semi-defined medium with glucose as carbon source passed through well-defined phases corresponding to trophophase, idiophase (enniatin production), and a degenerative phase. All the glucose was consumed by day 6 , at which time the mycelial dry weight had reached only half its maximum. When glucose was replaced by lactose, there was no separation of trophophase and idiophase. Enniatin production, dry weight, and sugar and nitrogen consumption were in approximate balance throughout the growth period ( 25 days), after which slow degeneration began. The term 'unitary metabolism' is proposed for this type of unphased behaviour. Unitary metabolism may approximate more closely to that occurring under natural conditions than does the metabolic phase separation observed when rapidly utilized carbon sources are used in laboratory cultures.
\end{abstract}

\section{INTRODUCTION}

It has often been observed that the growth and metabolism of fungi in submerged culture pass through well-defined phases. After inoculation the organism grows exponentially, in what is termed a balanced phase (Borrow et al. 196I) or trophophase (Bu'Lock, 1967); this is terminated by metabolic changes, and growth ceases. Thereafter, compounds with no obvious metabolic function may accumulate; these are called secondary metabolites, and the period of their formation is called idiophase (Bu'Lock, 1967). Biochemically, trophophase is thought to be a period of 'primary metabolism', during which synthesis of normal cell contents and walls occurs. It ends when an essential nutrient is exhausted (Bu'Lock, I967; Demain, 1968); precursors of normal cell materials then accumulate. There follows a period of 'secondary metabolism', originally termed shunt metabolism (Foster, 1949), during which these precursors undergo further reactions to form secondary metabolites. The term idiophase derives from the fact that secondary metabolites are usually species- or even strainspecific. The significance of secondary metabolism and its products was recently reviewed by Weinberg (1971).

The question of metabolic phases in static fungal cultures has received little attention. In Pithomyces cultures, species-specific cyclodepsipeptides (Russell, 1966) are detectable from an early stage (Done, Mortimer, Taylor \& Russell, 196I; Bishop et al. 1965), but since these compounds are located on the conidial surface (Bertaud, Morice, Russell \& Taylor, I963; Bishop et al. 1965; Riches, Rothwell \& Russell, 1967) their amount is a measure of sporulation rather than growth. In the present work, production of the chemically related enniatins (Gäumann et al. 1947) by Fusarium sambucinum Fuckel HLX316 (Audhya \& Russell, I $974 a, b$ ) was studied in relation to its growth and metabolism in liquid surface culture. A preliminary report has appeared (Audhya \& Russell, 1973a). 
Previously (Audhya \& Russell, I974a), we studied nutritional and environmental effects on enniatin production by $F$. sambucinum only in the mycelium, on the grounds that the $\mathrm{C} / \mathrm{N}$ ratios used were such that little or no antibiotic would be released into the medium. The present work has confirmed the validity of this procedure for enniatin production on media with high $\mathrm{C} / \mathrm{N}$ ratios.

\section{METHODS}

Sugars. Glucose (Difco) had $[\alpha]_{D}^{20}+53.9^{\circ}$ and lactose (Aldrich Chemical Co. Inc., Milwaukee, Wisconsin, U.S.A.) had $[\alpha]_{\mathrm{D}}^{20}+54.0^{\circ}$. Polarimetry was done with $10 \%(\mathrm{w} / \mathrm{v})$ solutions prepared $24 \mathrm{~h}$ previously. Paper chromatography of $500 \mu \mathrm{g}$ portions on Whatman No. 3 MM paper in ethyl acetate-pyridine-water (I0:4:3, by vol.) followed by spraying with aniline hydrogen phthalate (Partridge, I948) gave a single spot with glucose; lactose was contaminated with a trace of material that co-chromatographed with galactose.

Cultivation of organisms. The tryptone-salts medium ( $\mathrm{mg} \mathrm{N} / \mathrm{ml}$ ) and the general methods for liquid surface cultivation of $F$. sambucinum Fuckel HLX3 6 have been described (Audhya \& Russell, I974a).

Analytical methods. Mycelial dry weights and nitrogen were determined as before (Audhya \& Russell, I974a). Enniatins were determined spectrophotometrically as enniatin A (Audhya \& Russell, 1973b). Enniatins extracted from the mycelium were measured in a partially purified mixture of enniatin and neutral lipid (Audhya \& Russell, 1974a). Enniatins were extracted from the culture filtrates also. Thus, filtrate $(500 \mathrm{ml})$ was extracted twice with equal volumes of diethyl ether. The combined extracts were dried with $\mathrm{MgSO}_{4}$ and decolorized by filtration through charcoal (Norit A; $25 \mathrm{~g}$ ) that was then washed with ether $(200 \mathrm{ml})$. The combined effluents were evaporated to dryness. Chloroform $(50 \mathrm{ml} / \mathrm{g}$ residue) was added and the solution passed through a column of silica gel (J. T. Baker Chemical Co., Phillipsburg, New Jersey, U.S.A., catalogue no. 3405; 8 g/ml) that was further washed with chloroform ( $15 \mathrm{ml} / \mathrm{g}$ ). The residue obtained by evaporating the combined effluents to dryness in vacuo was analysed for enniatins. The method was checked by adding known amounts of enniatins to culture filtrates; recovery exceeded $95 \%$.

Sugars were determined in culture filtrates. The filtrate $(5 \mathrm{ml})$ was shaken for 10 min with Amberlite CG-50(H) (100 mg, 100 to $200 \mathrm{mesh}$ ), centrifuged, shaken with Amberlite $\mathrm{CG}-4 \mathrm{~B}(\mathrm{OH})$ (100 mg, roo to $200 \mathrm{mesh}$ ) and again centrifuged. The deionized supernatant fluid (Io $\mu 1$ ) was examined by paper chromatography as described above. When only one sugar was detected, it was determined in duplicate portions of the supernatant fluid (Hanes, 1929) by using a standard curve for that sugar. Glucose in the presence of lactose was determined by glucose oxidase (Saloman \& Johnson, 1959), lactose in the presence of glucose by methylamine (Malpress \& Morrison, 1949). $\mathrm{pH}$ was measured using a glass electrode.

\section{RESULTS}

Fusarium sambucinum was grown on a tryptone-salts-glucose medium, at concentrations of $0.1 \% \mathrm{~N}$ and I \% C, under the conditions shown by Audhya \& Russell (1974a) to be optimal for enniatin production from glucose. Periodical analysis of such cultures showed that they passed through clearly defined phases. Within 9 days after inoculation all glucose had disappeared from the medium, and growth, as measured by dry weight of mycelium, had ceased. On day 6 , when $96 \%$ of the glucose had been consumed, no enniatins were detected, but by day 9 production had begun. Enniatins accumulated in the mycelium for about 3 weeks, after which cell lysis began; this was evidenced by a decline in dry weight and 


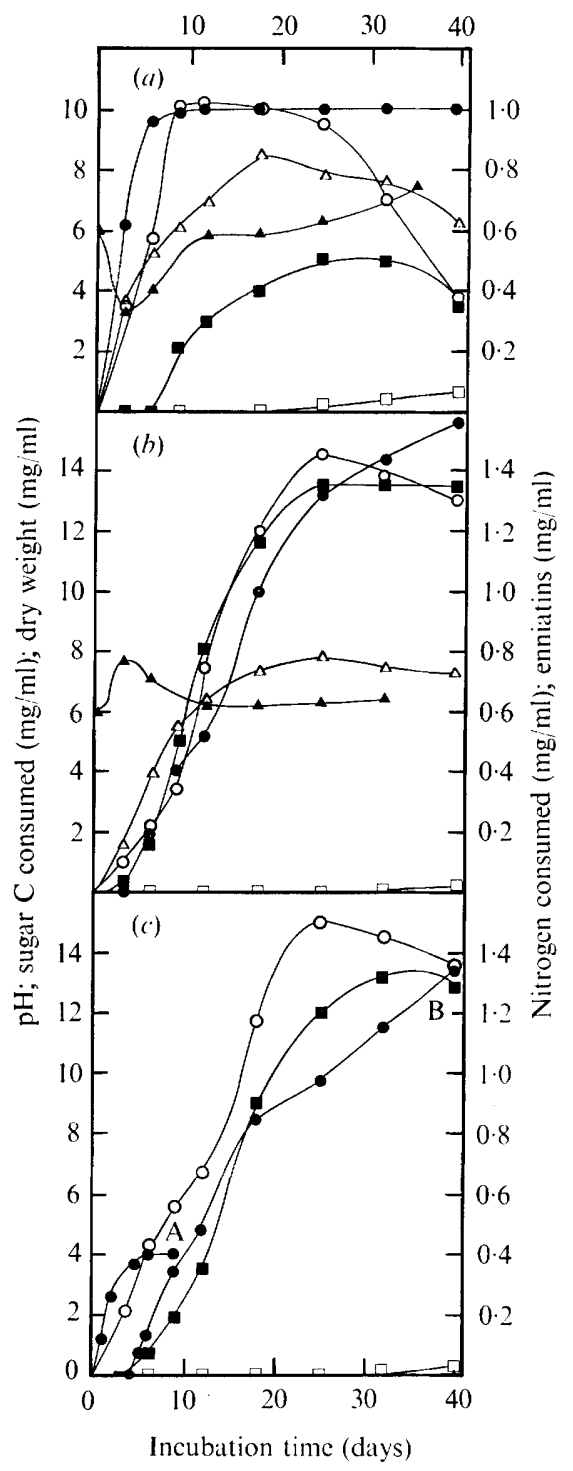

Fig. I. Fermentation parameters of $F$. sambucinum grown in surface culture in semi-defined media containing: (a) glucose ( $\%$, w/v, C); $(b)$ lactose $(2 \% \mathrm{C}) ;(c)$ glucose $(0.4 \% \mathrm{C})+$ lactose $(1 \cdot 6 \% \mathrm{C})$. The nitrogen source was tryptone $(0 \cdot 1 \% \mathrm{~N})$. Analyses are shown for dry weight $(\mathrm{O})$, sugar utilization $(\bullet)$, enniatins in mycelium $(\square)$ and medium $(\square)$, nitrogen utilization $(\triangle)$, and $\mathrm{pH}(\boldsymbol{\Delta})$. In $(c)$, $\mathbf{A}$ and $\mathbf{B}$ refer to glucose and lactose respectively.

a release of nitrogen into the medium (25 days). With accelerating lysis, the amount of mycelial enniatins declined and some antibiotic was released into the medium (Fig. I $a$ ). Thus, although some overlap occurred, periods corresponding to trophophase (o to 9 days), idiophase ( 6 to 32 days) and a degenerative phase ( 25 to 40 days) were clearly discernible.

Concurrently, and under otherwise identical conditions, cultures were grown without glucose but containing lactose in the concentration $(2 \% \mathrm{C})$ optimal for enniatin production from this sugar (Audhya \& Russell, 1974a). Growth, mycelial enniatin production, and nitrogen and sugar utilization occurred in concert (Fig. I $b$ ). We could detect no division 


\section{Table I. Economic coefficients for Fusarium sambucinum}

The organism was grown in surface culture at $20^{\circ} \mathrm{C}$ under standard lighting conditions (Audhya \& Russell, 1973c) on a semi-defined medium (Audhya \& Russell, 1974a) containing either glucose $(1 \%, w / v, C)$ or lactose $(2 \%, w i v, C)$ as carbon source.

\begin{tabular}{|c|c|c|}
\hline \multirow{2}{*}{$\begin{array}{c}\text { Time after inoculation } \\
\text { (days) }\end{array}$} & \multicolumn{2}{|c|}{$\begin{array}{c}\text { Economic coefficient } \\
\text { (g dry wt/g sugar consumed) on: }\end{array}$} \\
\hline & Glucose & Lactose \\
\hline 3 & 0.23 & -* \\
\hline 6 & 0.24 & 0.44 \\
\hline 9 & 0.41 & 0.35 \\
\hline 12 & 0.41 & 0.58 \\
\hline 18 & 0.40 & 0.48 \\
\hline 25 & 0.38 & 0.44 \\
\hline 32 & 0.28 & 0.38 \\
\hline 40 & 0.15 & 0.33 \\
\hline
\end{tabular}

into trophophase and idiophase during the 25 days of growth, and the subsequent degenerative phase was less marked than in cultures on glucose. At no stage did we detect any sugar but lactose in the medium.

A third, otherwise identical set of cultures contained as carbon sources glucose (10 $\mathrm{mg} / \mathrm{ml})$ and lactose $(40 \mathrm{mg} / \mathrm{ml})$ in a combined concentration corresponding to about $2 \%(\mathrm{w} / \mathrm{v}) \mathrm{C}$. Growth was rapid initially, and all the glucose was consumed by day 6 . Thereafter the cultures behaved like those containing lactose alone. Onset of enniatin production was delayed until the glucose was almost completely consumed, and the yield of enniatin was maximal about one week later than in cultures without glucose (Fig. I $c$ ).

Differences in the growth and production characteristics of $F$. sambucinum on glucose and lactose coincided with differences in metabolism (Fig. I $a, b$ ). On glucose, the economic coefficient (e.c. $=\mathrm{g}$ dry wt/g sugar consumed) was low in the early stages of growth, during which the $\mathrm{pH}$ of the medium decreased sharply. This suggests that the glucose was rapidly metabolized to organic acids. Thereafter both $\mathrm{pH}$ and e.c. rose, indicating utilization of the organic acids for growth. In cultures containing lactose alone, early growth (to 3 days) consumed no sugar and presumably utilized carbon from the amino acids of the tryptone; this would also account for the initial rise in $\mathrm{pH}$, due to liberated ammonia. After lactose consumption began, the $\mathrm{pH}$ of culture filtrates fell to the initial value of 6 , and c.c. values exceeded those attained in cultures containing glucose (Table 1 ).

\section{DISCUSSION}

The importance of carbon source in antibiotic production has long been recognized (Johnson, 1952) and has been thoroughly discussed by Demain (I968). Glucose may repress or inhibit antibiotic synthesis. This sugar is usually rapidly utilized in such a way that its acidic catabolites accumulate, an effect evident in Fig. I $(a)$. This can lead to catabolite repression, as illustrated by the synthesis of tryptophanase in Escherichia coli (Raunio, I966) and of phenoxazinone synthase in Streptomyces antibioticus (Gallo \& Katz, 1972). Repression or inhibition (Demain, 1968) of enzyme(s) needed for enniatin biosynthesis, or an unfavourable $\mathrm{pH}$ of the medium (Haavik, 1974), would explain the results shown in Fig. I $(a, c)$ : synthesis began only when most of the glucose and - perhaps more significantly - the acidic catabolites were largely consumed (Fig. I $a$ ). The lack of any lag in enniatin 
production from lactose might be similarly explained by slower sugar utilization; there was no accumulation of acidic catabolites (Fig. I $b$ ) or, judging from the relative constancy of the e.c., of appreciable amounts of any other metabolic intermediates. Similarly, Saltero \& Johnson (I953) obtained higher penicillin yields from lactose than from glucose, but equally good results without lactose when they periodically added small increments of glucose. Thus it appears that slow utilization of the carbon source promotes high antibiotic yields. Our own results prompt us to suggest further that the temporal separation of trophophase from idiophase may be a laboratory artefact, brought about by high concentrations of rapidly used carbon sources. In nature, fungi probably seldom encounter such conditions, and on their natural substrates may show what we propose to call 'unitary metabolism' of the type exhibited by $F$. sambucinum grown in static culture on lactose. The bacterium Bacillus licheniformis behaves similarly when producing bacitracin in submerged culture on glutamate (Haavik, 1974). We prefer the term 'unitary' to 'balanced', since balanced phase (Borrow et al. 1961) appears synonymous with Bu'Lock's (1967) trophophase - which, in our experiments with glucose, was distinguished by marked imbalance between nutrient utilization and dry-weight increase.

Bu'Lock (196I) suggested that secondary metabolism confers a selective advantage upon an organism, by maintaining the mechanisms essential to cell multiplication in operative order when growth is not possible. Our results with $F$. sambucinum on glucose are consistent with this hypothesis, but the results with lactose appear to negate it. Woodruff (I966) considered secondary metabolism a type of detoxication mechanism that disposes of accumulated small primary molecules in cells whose ability to remain in a state of balanced growth is diminishing. Again, our results with glucose are consistent with this viewpoint, but those with lactose inconsistent.

If 'secondary metabolism consists of the formation of chemically bizarre products following cessation of cell multiplication' (Weinberg, I97I), then $F$. sambucinum engages in secondary metabolism on glucose but not on lactose. Nevertheless, on lactose it forms the same 'chemically bizarre products', in greater amount and with more efficient sugar utilization for growth. This apparent contradiction strongly suggests that the 'function' of secondary metabolism (i.e. the presumed selective advantage conferred upon an organism by its formation of chemically bizarre products) still eludes us.

Since dry weight may be an inadequate index of growth (Taber \& Siepmann, 1965) and lactose may be a less 'natural' substrate than glucose, our findings do no more than suggest the occurrence of unitary metabolism under natural conditions. Nevertheless, we believe they are significant enough to warrant an examination of metabolism (growth, utilization of nutrients, and production of secondary metabolites) in this and other fungi under conditions as close as possible to those in nature. Solid-state fermentations for producing secondary metabolites (Done et al. 196I; Hesseltine, 1972) may provide a suitable approach to this problem.

This work, supported by grant MA-2480 from the Medical Research Council of Canada and an award (to T.K.A.) from the Killam Trust, was the subject of T.K.A.'s doctoral thesis. 


\section{REFERENCES}

Audhya, T. K. \& Russell, D. W. (1973a). Secondary metabolism-biological phenomenon or semantic quibble? Proceedings of the Canadian Federation of Biological Societies 16, 39.

Audhya, T. K. \& Russell, D. W. (1973 $b$ ). Spectrophotometric determination of enniatin A and valinomycin in fungal extracts by ion complexation. Analytical Letters 6, 265-274.

Audhy, T. K. \& Russell, D. W. (1973c). Production of enniatin A. Canadian Journal of Microbiology I9, $105 \mathrm{I}-\mathrm{I054}$.

AudhyA, T. K. \& Russell, D. W. (1974a). Production of enniatins by Fusarium sambucinum: selection of high-yield conditions from liquid surface cultures. Journal of General Microbiology 82, I 81-I90,

AUdhyA, T. K. \& RusselL, D. W. (1974b). Natural enniatin A, a mixture of optical isomers containing both erythro- and threo- $N$-methyl-L-isoleucine residues. Journal of the Chemical Society Perkin Transactions $\mathbf{r}$, $743-746$.

Bertacd, W. S., Morice, I. M., Russell, D. W. \& Taylor, A. (I963). The spore surface in Pithomyces chartarum. Journal of General Microbiology 32, 385-395.

Bishop, E., Griffiths, H., Russell, D. W., Ward, V. \& Gartside, R N. (i965). Spore surface depsipeptides in some Pithomyces species. Journal of General Microbiology 38, 289-297.

Borrow, A., Jefferys, E. G., Kessell, R. H. J., Lloyd, E. C., Lloyd, P. B. \& Nixon, E. S. (ig6r). The metabolism of Gibberella fujikuroi in stirred culture. Canadian Journal of Microbiology 7, 227-276.

Bu'Lock, J. D. (1961). Intermediary metabolism and antibiotic synthesis. Advances in Applied Microbiology 3, 293-342.

Bu'Lock, J. D. (1967). Essays in Biosynthesis and Microbial Development, pp. 49-58. New York: John Wiley.

DemaIN, A. L. (I968). Regulatory mechanisms and the industrial production of microbial metabolites. Lloydia 31, 395-4I8.

Done, J., Mortimer, P. H., TAylor, A. \& Russell, D. W. (I96I). The production of sporidesmin and sporidesmolides by Pithomyces chartarum. Journal of General Microbiology 26, 207-222.

Foster, J. W. (1949). Chemical Activities of Fungi, pp. 164-169. New York: Academic Press.

Gallo, M. \& KATZ, E. (I972). Regulation of secondary metabolite biosynthesis: catabolite repression of phenoxazinone synthase and actinomycin formation by glucose. Journal of Bacteriology ro9, 659-667.

Gäumann, E., Roth, S., Ettlinger, L., Plattner, P. A. \& Nager, U. (i947). Enniatin, ein neues, gegen Mykobakterien wirksames Antibiotikum. Experientia 3, 202-203.

HAAviK, H. I. (1974). Studies on the formation of bacitracin by Bacillus licheniformis: effect of glucose. Journal of General Microbiology 81, 383-390.

HANES, C. S. (1929). An application of the method of Hagedorn and Jensen to the determination of larger quantities of reducing sugars. Biochemical Journal 23, 99-106.

Hesseltine, C. W. (1972). Solid state fermentations. Biotechnology and Bioengineering 14, 517-532.

JoHnSON, M. J. (1952). Recent advances in penicillin fermentation. Bulletin of the World Health Organization 6, 99-I II,

Malpress, F. H. \& Morrison, A. B. (1949). The semi-micro estimation of lactose alone and in the presence of other sugars. Biochemical Journal $45,455-459$.

PARTRIDGE, S. M. (1948). Filter-paper partition chromatography of sugars. Biochemical Journal 42, $238-250$.

Raunio, R. (1966). The effects of pyruvate and related compounds on the induced formation of tryptophanase in Escherichia coli. Acta chemica scandinavica 20, I7-23.

Riches, P., Rothwell, A. \& Russell, D. W. (1967). The spore-surface depsipeptide of Pithomyces sacchari. Journal of General Microbiology 46, 77-83.

Russell, D. W. (I966). Cyclodepsipeptides. Quarterly Reviews of the Chemical Society 20, 559-576.

Saloman, L. L. \& Johnson, J. E. (1959). Enzymatic microdetermination of glucose in blood and urine. Analytical Chemistry 3I, 453-456.

Saltero, F. V. \& Johnson, M. J. (1953). The effect of carbohydrate nutrition on penicillin production by Penicillium chrysogenum Q-r76. Applied Microbiology 1, 52-57.

TABer, W. A. \& Siepmann, R. (1965). Measurement of growth in liquid cultures of molds. Applied Microbiology $13,827-829$.

Weinberg, E. D. (1971). Secondary metabolism: raison d'être. Perspectives in Biology and Medicine I4, $565-577$.

Woodruff, H. B. (1966). Biochemical studies of antimicrobial drugs. Symposia of the Society for General Microbiology 16, 22-46. 